\title{
Considering Intermittent Dormancy in an Advanced Life Support Systems Architecture
}

\author{
Miriam J. Sargusingh ${ }^{1}$ \\ NASA Lyndon B. Johnson Space Center, Houston, Texas, 77058 \\ and \\ Jay L. Perry ${ }^{2}$ \\ NASA George C. Marshall Space Flight Center, Huntsville, Alabama, 35812
}

\begin{abstract}
Many advanced human space exploration missions being considered by the National Aeronautics and Space Administration (NASA) include concepts in which in-space systems cycle between inhabited and uninhabited states. Managing the life support system (LSS) may be particularly challenged during these periods of intermittent dormancy. A study to identify LSS management challenges and considerations relating to dormancy is described. The study seeks to define concepts suitable for addressing intermittent dormancy states and to evaluate whether the reference LSS architectures being considered by the Advanced Exploration Systems (AES) Life Support Systems Project (LSSP) are sufficient to support this operational state. The primary focus of the study is the mission concept considered to be the most challenging - a crewed Mars mission with an extensive surface stay. Results from this study are presented and discussed.
\end{abstract}

\section{Nomenclature}

AES $=$ Advanced Exploration Systems

ECLSS = Environmental Control and Life Support System

$D S G \quad=$ Deep Space Gateway

$D S T=$ Deep Space Transport

$H E O=$ Human Exploration and Operations

LSS = life support system

$N A C \quad=$ NASA Advisory Council

NASA = National Aeronautics and Space Administration

\section{Introduction}

$I^{1}$

NTERMITTENT habitation of deep space exploration habitats is becoming a standard operational concept for crewed exploration missions. The most recent mission concepts presented at the National Aeronautics and Space Administration (NASA) Advisory Council (NAC) Human Exploration and Operations (HEO) Committee meetings in early calendar year 2017 show that exploration habitats could cycle between several weeks of human habitation and uninhabited (dormant) periods lasting a year or more. ${ }^{1,2}$ Though the Environment Control and Life Support System (ECLSS) is primarily designed to maintain a habitable, safe environment for the crew, it also provides important functions to support other vehicle systems during both active and dormant phases, ensures a safe, habitable environment upon the crew's return, and continues to operate through the next inhabited mission phase. While we strive to learn from our collective experiences during the Skylab and the International Space Station (ISS) programs, neither program has afforded us experience with intermittent dormancy on an closed loop ECLSS. Skylab experienced quiescent periods of one to three months between crewed periods. The ISS experienced similar duration quiescent periods during its early assembly yet during that time it lacked a full life support system and, therefore, depended on the

\footnotetext{
${ }^{1}$ Life Support Systems Engineer, Crew and Thermal Systems Division/EC3, 2101 NASA Parkway, AIAA Member.
}

${ }^{2}$ Lead Engineer, ECLS Systems Development Branch/ES62, Space Systems Department, AIAA Member. 
Shuttle orbiter for core ECLSS functions. This early review of the exploration ECLSS reference architecture will aid in identifying potential limitations and requirements for system automation. The following provides an overview of an early study of dormancy-related challenges and presents pertinent results for future LSS development.

\section{Dormancy Study Objectives and Scope}

The purpose of this study was to evaluate the implications of intermittent dormancy on the reference exploration ECLSS architecture. ${ }^{3}$ Representative technologies for each functional element were evaluated to provide a basis for understanding operational modes and dormant considerations. It is important to note that there are on-going development efforts in several ECLSS functional areas; the technologies chosen for these basis of this study are not indicative of a baseline or preferred design. The mission scenario evaluated as part of this study was derived from the March NAC HEO committee meeting. ${ }^{1}$

\section{A. Exploration Mission Scenarios}

The NASA has adopted a 3-phased approach to deep space exploration. The first phase involves build-up of the deep space transportation capability in cis-Lunar space with buildup of a Deep Space Gateway (DSG) and delivery of the Deep Space Transport (DST). The second phase involves assembly and checkout of the DST. The third phase involves deep space human exploration, kicked off with a crewed Mars orbital mission. The mission plan is visualized in Fig. 1.

Both the DSG and DST will undergo a cycle of crewed and quiescent phases. The crewed and dormant periods derived from the NASA published mission concepts are shown in Fig. 2 with the DST habitat reflected in the upper timeline and the DST habitat reflected in the lower timeline. The DSG will support approximately one month of habitation and is left dormant until the next crewed mission. Missions appear to occur about every year.

The DST is a reusable vehicle for shuttling crews to Mars. The general cycle would consist of a "refit" period during which maintenance is performed while attached to DSG in cis-lunar space, likely followed by a period of dormancy until the next mission crew arrives. The DST would then separate from the DSG to embark on a deep space mission. The first mission in the 2030s being a Mars orbital mission during which the DSG ECLSS must support the crew continuously for about three years. A future mission is also considered in which the DST is used to transport the crew to Mars, remains dormant in

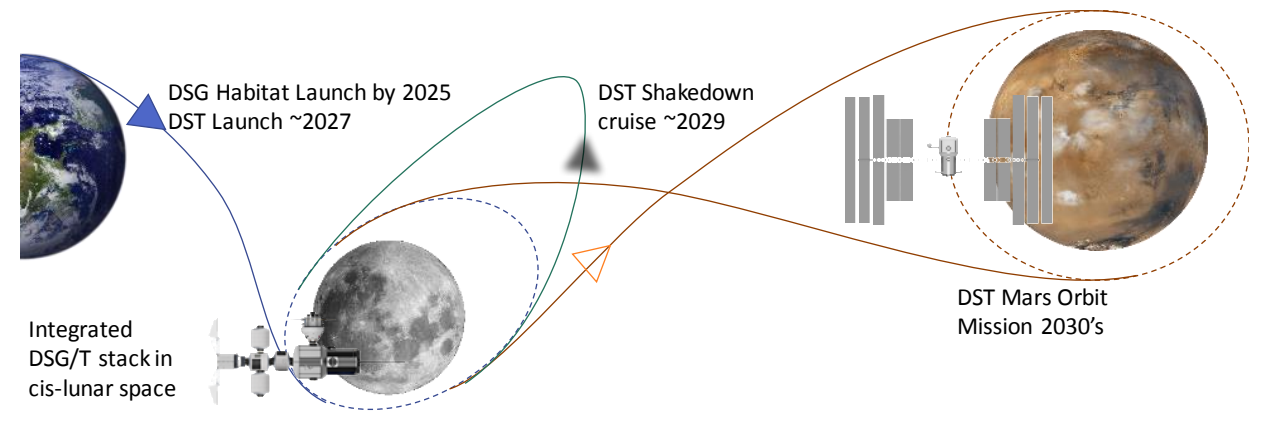

Figure 1. Visualization of the exploration mission plan.

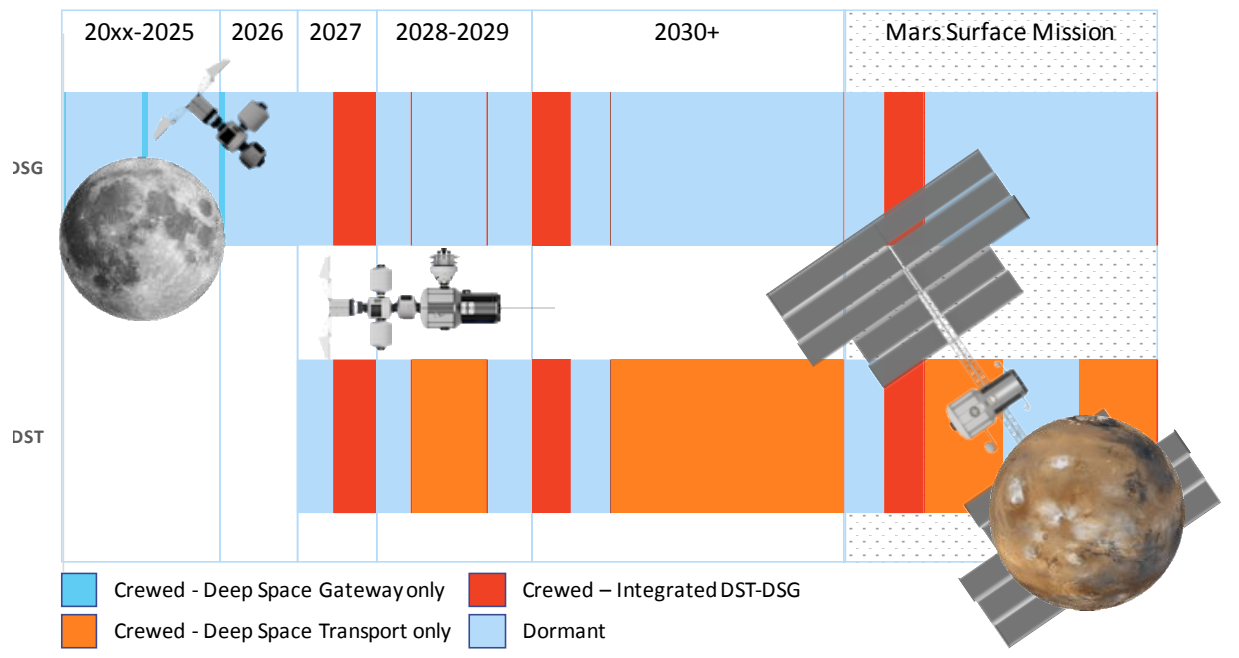

Figure 2. Exploration mission timeline. The timeline is extrapolated from the Human Exploration Plans presented to the NAC HEO Committee. ${ }^{1,2}$ The final "Mars Surface Mission" phase depicts a notional mission phase for the purposes of studying all aspects of ECLSS dormancy. 


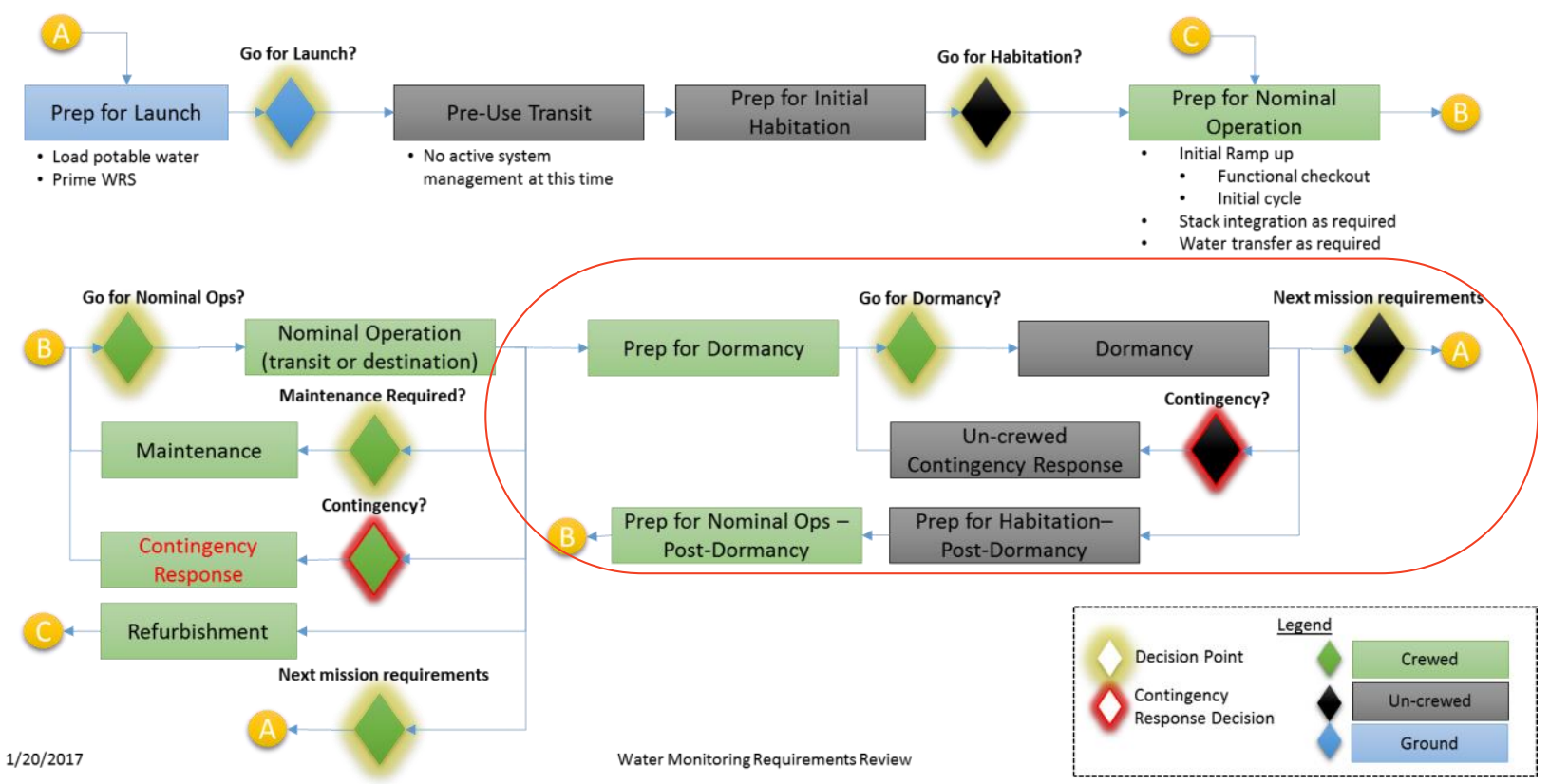

Figure 3. Generic exploration habitat mission flow diagram.

Mars orbit while the crew performs a yearlong surface mission, and then is re-inhabited for a one year return trip to dock with the DSG in cis-lunar space.

Figure 3 shows the generic mission flow diagram for an exploration habitat. This study focuses on the intermittent transient activities associated with dormant periods reflected in the red oval rather than the dormant period experienced by the system prior to initial habitation on orbit. Dormancy phases include preparation and transition to the dormant state, system maintenance during the quiescent period, and transition out of the dormant state.

During the dormant periods, the ECLSS must maintain a threshold atmosphere to protect itself and other vehicle systems. This includes maintaining a minimum pressure and temperature to protect against freezing, and controlling humidity to protect against condensation. The ECLSS must be capable of guaranteeing a habitable atmosphere prior to crew return by providing an acceptable total pressure, oxygen partial pressure, trace gaseous contaminant levels, and temperature. Part of this is being able to detect and resolve anomalous conditions autonomously. During ramp-up or "Prep for Nominal Ops", full recovery and verification of ECLSS functionality must be attained to support the next crewed mission phase. The full ramp-up sequence may involve autonomous activities prior to crew return and additional activities performed by the crew upon their return.

\section{B. Exploration Life Support System Architecture}

\section{Deep Space Gateway}

The DSG will include the habitat, an airlock, and logistics modules. ${ }^{1}$ It is assumed to have a mostly open-loop ECLSS that can support a crew of four for up to 30 days (not including contingency). Figure 4 shows the ECLSS functionality for a DSG habitat which provides five basic environment control functions - pressure control (depicted as "2-gas Control"), carbon dioxide $\left(\mathrm{CO}_{2}\right)$ removal, trace contaminant

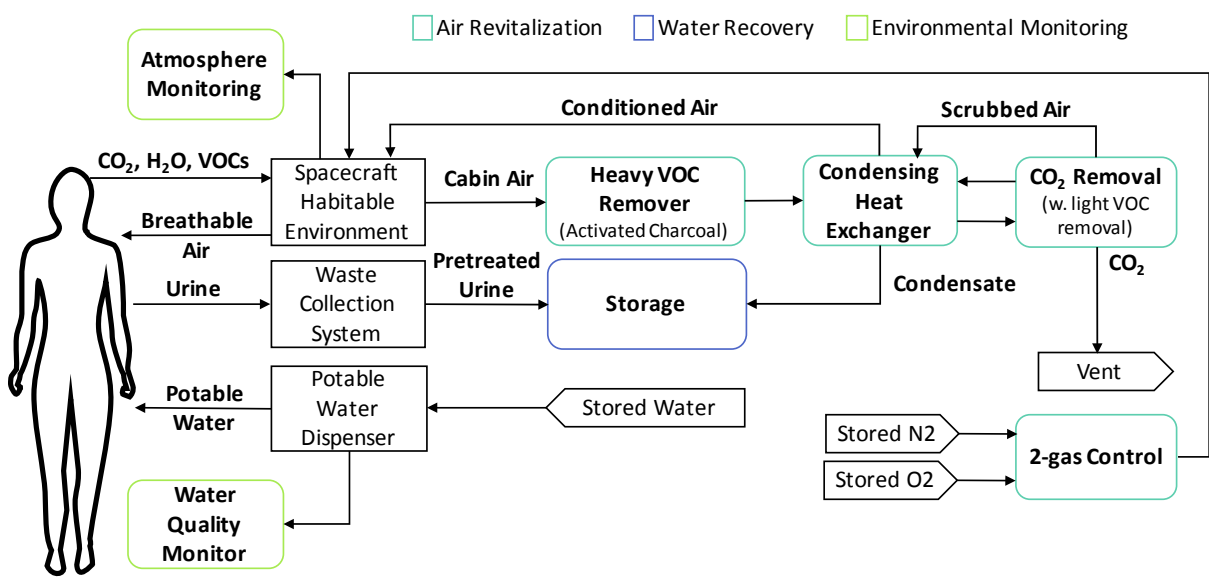

Figure 4. DSG reference ECLSS architecture.

3

American Institute of Aeronautics and Astronautics 
control, temperature and humidity control, and environmental monitoring. Environmental monitoring includes cabin atmospheric composition, water quality monitoring, and emergency condition monitoring. In addition, the ECLSS will coordinate resources to support airlock suit operations. Any wastewater that may be collected will be stored for disposal or processing by a water recovery system in another asset (the DST or other internationally-provided habitat). Potable water, nitrogen and oxygen are consumables to be supplied to support DSG crewed operations. The Orion, or other transport vehicle, is always present when the DSG is inhabited and may provide some of the ECLSS functionality, e.g. the Waste Collection System, as well as functional redundancy.

\section{Deep Space Transport}

The DST is assumed to comprise a long-duration closed loop ECLSS more closely resembling that of the ISS. It expands on the functions of the DSG habitat to include all functionality independent of any other vehicle, as well as regenerable closed-loop functionality including water recovery, oxygen generation, and carbon dioxide reduction as shown by Fig. 5 .

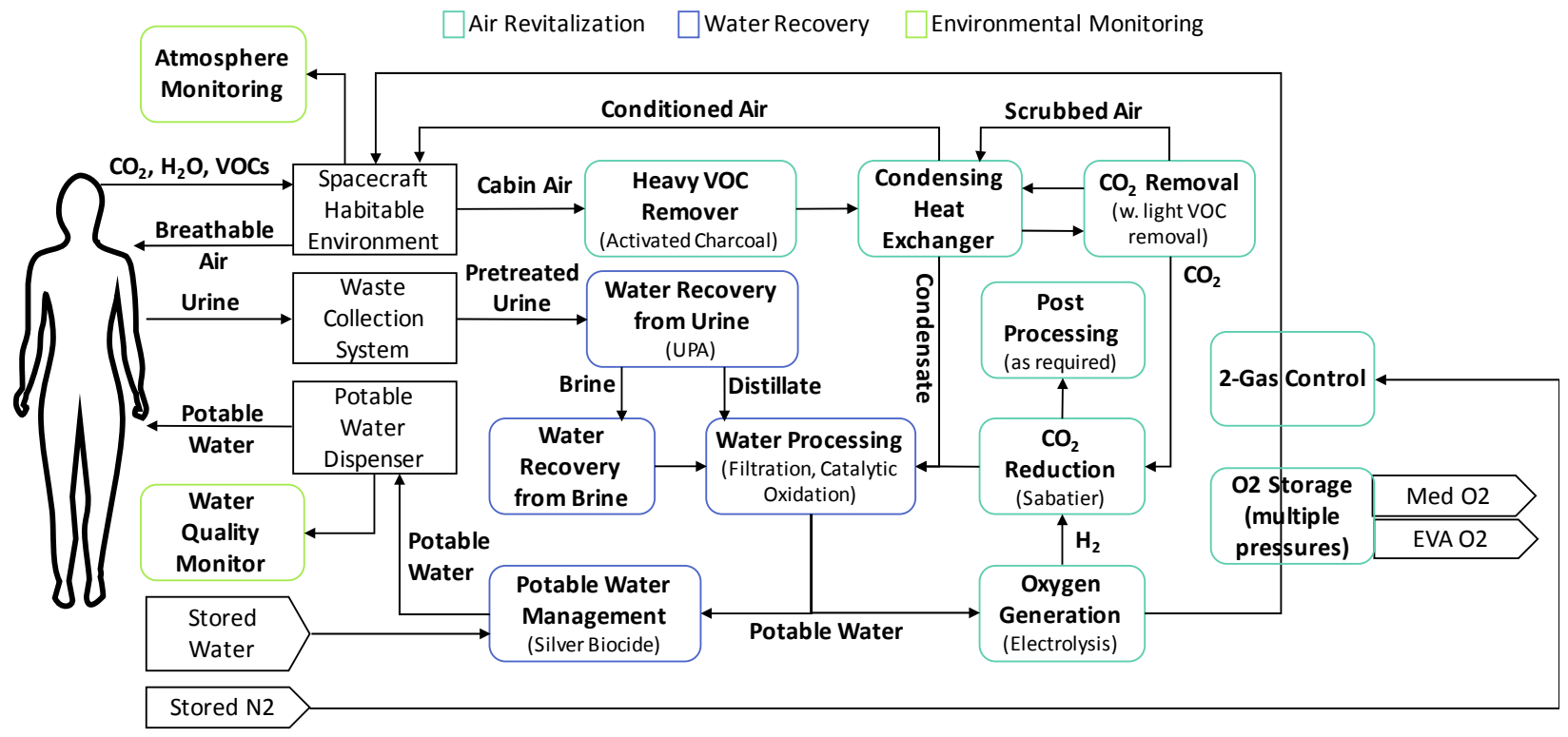

Figure 5. DST reference ECLSS architecture.

\section{Concept of Operations for Dormancy}

The spacecraft LSS provides minimal capability during uninhabited quiescent mission phases or dormant periods. The required functions are limited to maintaining an environment in the habitat's pressurized volume that is sufficient to support the vehicle systems. Environmental parameters to be considered for dormant period maintenance include temperature, pressure, humidity, and $\mathrm{O}_{2}$ partial pressure levels. It is expected that the control bands will be much wider than for crewed phases. Insight into system health and consumables quantities during dormancy will be key to ensure the safe return of the crew from Mars orbit.

\section{A. Preparing for Dormancy}

The transition to dormant operations for the LSS will center on allowing the elements to remain in a standby state until the transition out of dormancy is initiated. Some functions will need to be maintained through the transition period in order to support the crew until they fully transition to another vehicle. Modules should be isolated with intermodule ventilation (IMV) activated in order to minimize propagation of catastrophic events such as a fire or a nonrecoverable pressure leak. The resultant dormant ECLSS configuration is shown in Fig. 6.

1. Atmosphere Conditioning

Nominal atmospheric conditions will be maintained until the crew is fully transitioned to the other vehicle. Nonessential systems, such as the carbon dioxide removal system, will be turned off once the crew has departed the habitat. Heaters and ventilation will remain active in the dormancy operational mode throughout the dormant phase. Relative humidity will be drastically reduced during the dormant period which may require continued operation of ECLSS systems for a period of time after the crew departs to achieve the desired level. 


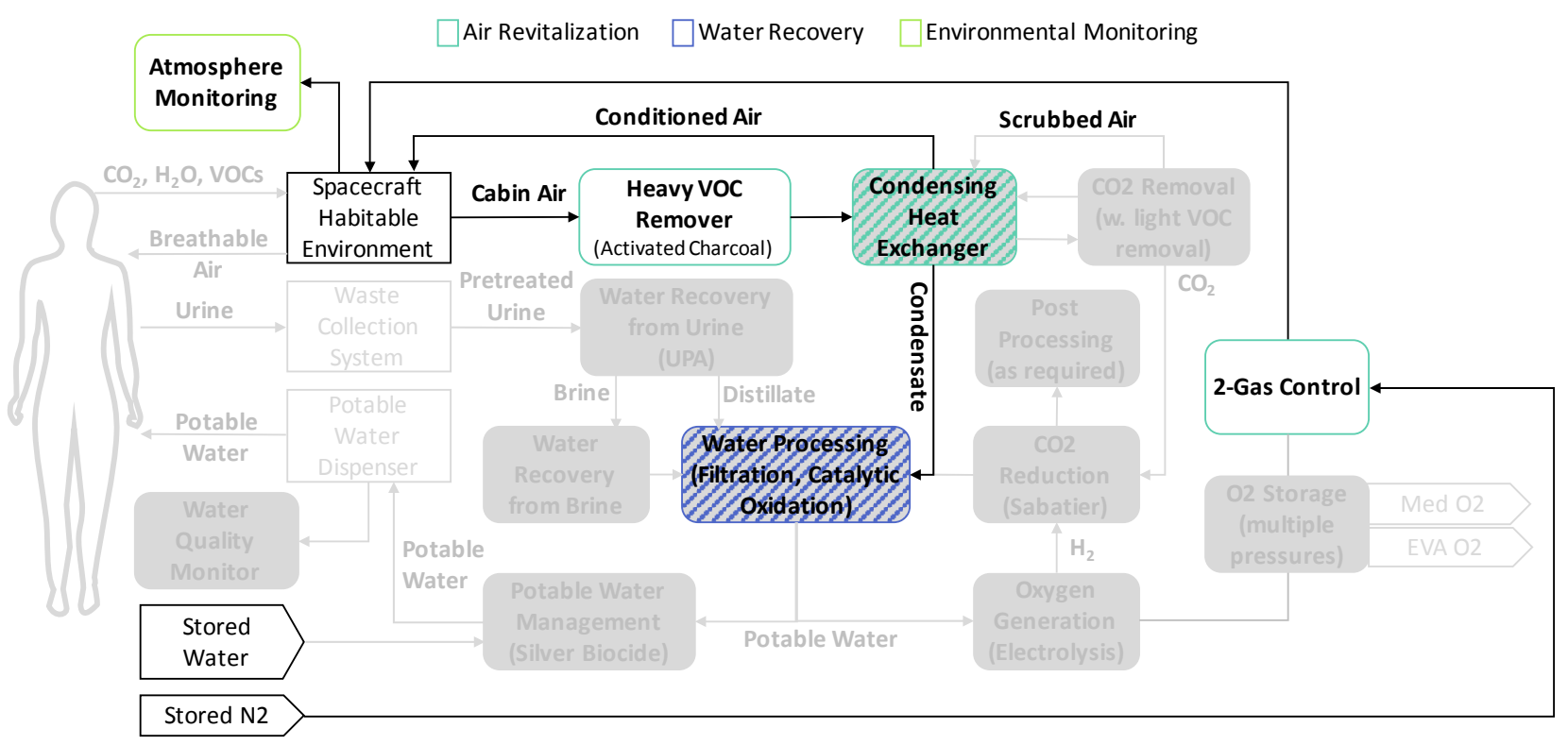

Figure 6. AES LSS dormant configuration. The diagram depicts the closed-loop ECLSS dormant configuration consisting of pressure control with nitrogen only, atmospheric monitoring, some level of trace contaminant and humidity control. Though not explicitly shown, the cabin/conditioned air loop includes ventilation and thermal control.

\section{Pressure Management}

Pressure management will be placed in a standby mode in which nitrogen is used to maintain a lower total pressure. Disabling the oxygen flow into the cabin is recommended during dormancy to reduce the likelihood of fire in the cabin. The minimum pressure is to be defined and will be derived from other vehicle system sensitivities. Pressure and temperature will need to be balanced such that systems containing water do not freeze.

3. Water Management

Draw down of nominal water stores will occur ahead of a dormant period to reduce the amount of water remaining in the system. Where possible, water stores should be segregated from water recovery systems and the contaminated portions of the dispensing system. Water recovery systems will be flushed and treated with biocide prior to dormancy to minimize the biological contaminants in the system and then placed in a standby mode for the duration of the dormant period.

4. Monitoring

Environmental monitoring will remain active during the dormant phase. As mentioned previously, sufficient ventilation will be provided to support adequate mixing and sampling during dormancy.

\section{B. Transition Out of Dormancy}

Prior to crew return, a habitable environment must be established. This involves repressurizing the habitat, restablishing a sufficient oxygen partial pressure, engaged the trace contaminant control system to remove offgassed products that accumulated during the dormant period, and using the heaters to raise the temperature. A humidifier which is not currently in the architecture may be necessary to raise the humidity to required levels.

Prior to nominal operations, the remaining ECLSS assemblies will need to be activated and verified functional. The water recovery and management system will need to be returned to its operational configuration. The potable water system may need to be recycled to ensure adequate and consistent water quality.

\section{Driving Considerations for Life Support System Dormancy}

Operations relating to ECLSS dormancy must consider aspects concerning maintaining cabin conditions during the quiescent period and recovering the ECLSS functions when the crew returns. Awareness and preparation for contingency operations must be included in these considerations to ensure crew safety through the next mission phase. Some key challenges identified in the study are as follows. 


\section{A. Microbial Growth Control}

Controlling microbial growth in crewed spacecraft is necessary to protect the health of the $\mathrm{crew}^{4}$, this is particularly so during intermittent dormant periods after the habitat and ECLSS have been subjected to organic matter from the crew and other biological experiments/systems. General mitigation strategies include regular disinfection and cleaning, changing the environment to be less favorable to growth, and limiting free air-water interfaces. ${ }^{4}$

Technology development efforts have been underway to extend on-orbit microbial detection and speciation capabilities, to identify additional disinfection techniques such as ultraviolet lights ${ }^{5}$, and incorporating anti-microbial coatings in systems like the condensing heat exchanger that are normally subject to condensation.

\section{Temperature and Humidity Control}

One challenge identified during this initial study was a balance between thermal and humidity control. This stems from the initial goal of manipulating the environment to inhibit microbial growth by reducing the temperature. ${ }^{5}$ Without the crew present, the minimum spacecraft environmental temperature is driven by the remaining vehicle systems; i.e. we cannot go below freezing. The challenge is that the reduced temperature will cause humid air to condensate on the cold surfaces causing free liquid in the habitable volume that can damage vehicle systems as well as host microbial growth.

To mitigate this consequence the cabin atmosphere must be dried before reducing the cabin temperature. The approach developed by the ISS Program when considering de-manning is to leave the condensing heat exchanger active at a low coolant temperature after the crew departs to achieve the desired humidity level. ${ }^{6}$ With air continuously being moved through the condensing heat exchanger during the dormant phase, the condensation surfaces will be dried. However the pump and fluid line to the condensate storage vessel would remain vulnerable to microbial growth. Other options to humidity control that are being evaluated include using a consumable desiccant, as was done during ISS early assembly stages, or using other regenerable contaminant sorbent systems such as the Orion $\mathrm{CO}_{2}$ and Moisture Removal Amine Swing-bed (CAMRAS).

Once the appropriate temperature and humidity levels are achieved, shell heaters will be used to maintain the targeted temperature. Ventilation will be active throughout the dormant phase to ensure sufficient air mixing and thermal distribution so there are no localized cold, damp areas. Cabin atmosphere composition monitoring will also be active to verify appropriate humidity levels.

A challenge to humidity reduction lies in the ability to recover a habitable environment. The ECLSS architecture must to be updated to include a means to humidify the cabin prior to the crew's return, or shortly after. Another challenge lies in potential impacts to vehicle avionics due electrostatic discharge control. Further work with avionics subject matter experts must be performed to understand the risks and potential impacts.

\section{Potable Water Quality}

The crew interface to the water management system is the most vulnerable to microbial growth. The dispensing needle should be removed and the crew interface area cleaned prior to dormancy. Upon return, the potable water dispenser should be flushed (reprocessing the water in the DST) and tested prior to allowing the crew to consume the water. $^{7}$

\section{B. Biomass Growth in Wetted Components}

All of the ECLSS elements that contain water are susceptible to biological growth, including the wastewater processing and management systems, and the oxygen generator. ${ }^{7}$ Biofilms and masses can block flow passages and coat reaction surfaces to reducing system performance or preventing operation.

\section{Water Processing Systems}

Flushing wastewater lines with treated, clean water can reduce contaminants and mitigate significant biological growth during dormancy. A biocide would be used to prevent biological growth in the potable water storage and distribution systems. Silver biocide currently considered as baseline for the AES ECLSS architecture becomes depleted over time and must be regularly replenished to maintain its efficacy which means this system may need to be active during dormancy. Further work is required to refine the water disinfection strategy and evaluate dormancy operations.

\section{Oxygen Generation}

Electrolysis cells used to convert water to hydrogen and oxygen is not compatible with chemical biocides which may leave a good portion of the oxygen generator vulnerable to biofilm growth during long duration periods of stagnation. Suitable options for controlling microbial growth have not been identified. Forward work includes review of past experience with operating ground units that have undergone long periods of intermittent dormancy. Targeted dormancy testing should also be performed to understand the vulnerabilities of the oxygen generator. 


\section{Risk of Long-term Hardware Inactivity}

Long-term hardware inactivity also presents a risk. Materials degradation and deformation may cause damage to seals allowing them to leak. Rotating equipment is susceptible to seizing after long periods of inactivity. Sensor calibration may also drift over time and may potentially be impacted by the different pressure and temperature environment during the dormant phase. These issues must be taken into consideration during the design, development, test, and evaluation phase of the DSG/DST ECLSS development to ensure each component can survive the dormant periods. Hardware may be periodically cycled during the dormant phase to mitigate these effects. The ability to remotely calibrate critical sensors may be necessary.

\section{Contingency Response Considerations}

Upon return, the crew may be faced with addressing contingencies which may threaten safety and habitability. Several areas for contingency response considerations are the following:

1. Atmosphere Conditioning

The atmosphere revitalization system will be in a stand-by mode during dormancy. Failures could occur during pre-habitation ramp-up operations. Controller faults may be cleared remotely. Hardware faults would have to be resolved by the crew wearing the appropriate emergency personal protective equipment (PPE) which may be gloves, goggles, and a dust mask at a minimum and a suit or emergency breathing mask under more dire circumstances. A failure of the IMV system, which should be active during dormancy, would lead to poor air mixing and invalidate telemetry received from the environmental monitors. In this case, the crew would have to assume a non-habitable environment and don PPE for troubleshooting and recovery activities.

Cabin atmosphere contamination leading to a hazardous accumulation and/or oxygen deficiency may be indicated by fire detection and suppression (FDS) and system chemical leak monitoring, e.g. thermal working fluid or other chemical fluid leaks. The FDS and system chemical monitoring may return false indications such that confirmation via environmental monitoring and system status evaluation means must be conducted. Remote cabin atmosphere sampling capability may be employed to confirm the nature and magnitude of the contamination. Entry of the affected volume requires PPE and well-established ventilation. Care must be taken to avoid cross-contamination of the docked vehicle habitable cabin during entry operations.

Some systems, such as the oxygen generator, have inherent hazards that could lead to loss of the habitat. Hazard controls will have to be implemented into the design of these systems. These systems should not be operating during dormant periods. During ramp-up, these systems should not be initiated unless telemetry and remote command capability is established.

\section{Pressure Management}

A failure of the pressure management system during remote ramp-up would lead to a non-habitable environment. The crew may be required to don breathing masks or pressurized suits for troubleshooting and recovery activities.

\section{Water Management}

Water Management system would be as dry as possible during dormancy. A leak of water may be detected by continuous monitoring of the tank levels, and cabin humidity. Biological growth may be detected with in-line monitoring, and/or sample analysis by the crew upon return. Manual troubleshooting, repair \& recovery may be performed by the crew upon return provided sufficient stored water.

\section{Waste Management}

Waste management systems should be as dry as possible during dormancy. A leak of wastewater may be detected by continuous monitoring of the tank levels and cabin humidity. A leak of urine pre-treatment chemicals or pretreated urine can lead to corrosion of contacted systems; therefore, additional monitoring may be required. Manual troubleshooting, repair, recovery, and clean-up may be performed by the crew wearing the appropriate PPE.

\section{Environmental Monitoring}

In the case of a failure of the environmental monitoring system, the crew would have to assume a non-habitable environment and don emergency equipment for troubleshooting and recovery activities.

\section{Conclusion and Forward Work}

Long duration intermittent dormancy presents challenges to the ECLSS that have not been experienced in previous crewed spacecraft programs. Of critical concern is control of biological growth, particularly in systems with no active microbial control like the oxygen generator. Further evaluation and testing must be performed to understand the impacts of long term dormancy on these systems. Concepts for re-establishing a habitable environment prior to crew return must be refined and demonstrated. Review of the dormant induced temperature, pressure, and humidity environment must be reviewed with the other vehicle systems to understand impacts and risks. 


\section{Acknowledgments}

The authors are pleased to acknowledge the contributions of the AES Life Support Systems Project management, Walter Schneider and Sarah Shull. Analytical support was provided by the AES LSS Modeling and Simulation Team Imelda Stambaugh, Greg Schunk, Bruce Conger and Glenn Waguespack. Technology leads Layne Carter, Mike Callahan, Scott Hanson, and Jeff Sweterlitsch providing insight into the emerging systems. ISS Program representatives provided insight in past experiences: Brienne Shkedi, Jason Dake, and Chris Brown. Other stakeholders and subject matter experts consulted during the course of this study included Molly Anderson, Mark Ott, Gene Unger, and Jim Broyan.

\section{References}

${ }^{1}$ Williams, G. "Human Exploration Plans". NASA Advisory Council Human Exploration and Operations Committee Meeting, Hampton, VA, July 24-25, 2017.

${ }^{2}$ Gerstenmeier, B. "Progress in Defining the Deep Space Gateway and Transport Plan." NASA Advisory Council Human Exploration and Operations Committee Meeting, NASA Headquarters, Washington D.C., March 28, 2017.

${ }^{3}$ Perry, J. L, Howard, D., Toomarian, N., Sargusingh, M. J., "Notional Environmental Control and Life Support System Architectures for Human Exploration beyond Low-Earth Orbit." AIAA 2016-5641, AIAA SPACE Forum, Pasadena, CA, Aug. 31 - Sep $2,2015$.

${ }^{4}$ NASA Space Flight Human-System Standard, Volume 2: Human Factors, Habitability, and Environmental Health, NASASTD-3001, Feb. 10, 2015

5 "Spacecraft Operations during Uncrewed Dormant Phases of Human Exploration Missions Capability White Paper", Oct. 8, 2015.

${ }^{6}$ Flight Rule B2-152, “Crew Contingency Return”, Aug. 9, 2016.

${ }^{7}$ Carter, D. and Tabb, D., "Water Recovery System Design to Accommodate Dormant Periods for Manned Missions, ICES2015-75, 45 ${ }^{\text {th }}$ International Conference on Environmental Systems, Bellevue, Washington, 2015. 